\title{
Integrated scheduling of tasks and gynecologists to improve patient appointment scheduling; a case study
}

\author{
N.M. van de Vrugt ${ }^{\mathrm{a}, \mathrm{b}, *}$, S.T. Luen-English ${ }^{\mathrm{c}}$, W.A.P. Bastiaansen ${ }^{\mathrm{b}}$, S. Kleinluchtenbeld ${ }^{\mathrm{b}}$, \\ W.T.P. Lardinois ${ }^{\mathrm{b}}$, M.H. Pots ${ }^{\mathrm{b}}$, D.J. Schoonbergen ${ }^{\mathrm{b}}$, E.W. Hans ${ }^{\mathrm{b}}$, J.L. Hurink ${ }^{\mathrm{b}}$, \\ R.J. Boucherie ${ }^{b}$ \\ a Jeroen Bosch Hospital, 's Hertogenbosch, The Netherlands \\ ${ }^{\mathrm{b}}$ Center for Healthcare Operations Improvement and Research (CHOIR), University of Twente, Enschede, The Netherlands \\ c School of Mathematics, Cardiff University, Cardiff, Wales, United Kingdom
}

\section{A R T I C L E I N F O}

\section{Article history:}

Received 23 December 2016

Accepted 27 November 2017

Available online 8 December 2017

\section{Keywords:}

Physician scheduling

Integer programming

Heuristics

Health care

\begin{abstract}
A B S T R A C T
Like many hospital departments, the gynecology department of the Jeroen Bosch Hospital experienced difficulties with scheduling outpatient appointments at the medically preferred times. Despite the time invested in creating the schedule, the compliance of the achieved schedules with the preferences of gynecologists is low, and the number of scheduled outpatient clinic hours is unbalanced over the weeks. To overcome this unbalanced scheduling, we develop a scheduling methodology that, opposed to existing methods, simultaneously assigns task types and gynecologists to shifts. This enables us to (1) explore the complete solution space to obtain better schedules, and (2) take into account different specializations and working hours of the gynecologists. To this end, we first present a Mixed Integer Linear Programming (MIP) approach for this scheduling problem that has the objective to increase compliance of the soft constraints. Preliminary results achieved with this MIP model show the potential of the chosen approach and were the motivation to develop two heuristic approaches, which are better suited for practical purposes. Based on several realistic test instances, the scheduling approaches appear promising for the hospital to apply for gynecologist scheduling, as they improve patient access times, comply better with preferences of the gynecologists, significantly reduce the time spent on creating the schedules, and do not require MIP solvers.
\end{abstract}

(C) 2017 Elsevier Ltd. All rights reserved.

\section{Introduction}

The gynecology department of the Jeroen Bosch Hospital (JBH) experienced difficulties with scheduling outpatients appointments at the medically preferred times. The problem is that pregnant women typically have fixed intervals between two visits to the gynecologist, while the offered number of consultation hours varies from week to week. A gynecologist has, like all other health care professionals, many different tasks in a hospital. Next to seeing patients at the ward, a specialist should be present in the operating theater and outpatient clinic, and leave for study purposes like attending conferences. Making a schedule for these specialists is therefore a difficult job, at the JBH currently performed by one of the gynecologists. This specialist spends two days each six week

\footnotetext{
* Current employed by: Leiden University Medical Centre, Leiden, The Netherlands.

E-mail address: n.m.vandevrugt@utwente.nl (N.M. van de Vrugt).
}

period to determine an adequate schedule manually, and additionally spends much time to make alterations to the schedule on request of colleagues.

When creating a schedule, the scheduler has to assign many tasks of different types to shifts, all with their own requirements regarding for example task frequency. Additionally, the scheduler has to assign gynecologists to the available shifts in the schedule such that they are able to perform all the tasks assigned to the shifts. The challenges are that there are many constraints on the allowed assignments, e.g., all tasks have different requirements, and some tasks may only be scheduled in a series with other tasks. Both the gynecologists and manager of the outpatient clinic see room for improvements in the schedules; gynecologists prefer more compliance with their preferences, and the manager of the outpatient clinic prefers a more balanced number of outpatient clinic hours throughout the weeks. Additionally, the time to create the schedules should be reduced significantly as this reduces costs, allows the scheduler to spend more time on patient care, and gives more flexibility in the scheduling process as several possible schedules could be compared quickly. 
The literature on workforce scheduling is quite abundant, and in the following we will highlight some related research. There are two recent reviews on workforce scheduling: a general review [1], and a review focused on incorporating skill levels of the workforce [2]. From the latter, we conclude that there are several papers that investigate both task and employee scheduling, of which some incorporate non-hierarchical skill-levels of the employees. However, none of these papers incorporates preferences of the employees in the model. A large part of the literature in this field focuses on scheduling a list of tasks, which have specific timewindows in which they may be scheduled, to an employee such that the workload does not exceed one workday or shift, cf. [3]. This problem differs from the gynecologist scheduling problem, since the continuity of certain tasks (i.e., balancing the occurrences of tasks between workweeks) is not a major issue when scheduling a task list.

A different, but related field of literature considers scheduling employees to predefined repetitive shifts, for example two employees always have to be scheduled in the night shift. Most papers in this field present integer programming models that are too large to solve in a reasonable amount of time, and to overcome this problem partial branch-and-bound [4], branch-and-cut [5], TABU search [6] and simulated annealing [7] approaches are taken. In [8] the solution is approximated by splitting the model into many small sub-models. Other approaches taken are constraint programming $[9,10]$ and goal programming [11]. Of these papers, only [8] consider employees with different skill-sets. In these papers the workload is balanced fairly over all employees. In the current paper, we additionally balance the number of scheduled tasks of each type over the weeks.

Gunawan and Lau [12] consider the problem of assigning medical specialists to different duty types, which have limitations on the number of duties of the same type that can be performed in the same shift (due to a limited number of resources such as treatment rooms). The authors focus on ergonomic constraints, such as the distribution of physically heavy duties, to ensure a fair schedule. The main difference with the current research is that employees' specializations are not incorporated, and, due to the scheduling horizon of one week, the model can be solved to optimality.

In a recent paper, Fügener et al. [13] create a duty schedule for an anesthesiology department. The authors invoke a mixed integer programming approach to first assign shifts to the specialists, and then specialists to workstations. Job qualification levels are incorporated, and are increasing in the number of years of experience and education of the anesthesiologists. The two stage scheduling approach is also applied in $[8,14]$, to obtain a workforce schedule for air force meal service employees and general service employees, respectively. In these papers, heuristics are used to solve the large integer linear programs that are constructed to optimize the schedules. The problem considered in the current paper differs from all these papers, as each gynecologist has a (different) subset of tasks she is allowed to perform, and some highly specialized tasks may only be performed by one gynecologist. As a consequence, we do not have the option to schedule an 'overqualified' gynecologist, which is possible when job qualification levels increase with hierarchy levels. Erhard et al. [15] provide a literature review, to which the reader is referred for additional related references.

In this paper we focus on a case study performed at the gynecology department of the JBH, which is clarified in the following section. To get more insight in the scheduling of tasks and gynecologists at the JBH, we develop a mixed integer linear program (MIP) approach, which aims to optimize a weighted sum of soft constraint compliance. In this model we simultaneously assign tasks and gynecologists to shifts, thereby deviating from a sequential decision making. The reason for choosing a simultaneous decision is clarified in the following section, where we provide an example why a combined assignment of tasks and gynecologists to shifts is preferable. This example shows that, compared to models with sequential decision-making, our approach can better incorporate (1) specializations of the gynecologists that are not strictly increasing in hierarchy levels, and (2) different working hours of the gynecologists. Hereby, the requirements of the different task types have to be taken into account, which implies that each task of a certain type may only be assigned to a subset of the gynecologists. The MIP takes a prioritized list of hard and soft constraints into account, which is determined by the gynecologists and the outpatient clinic manager. The outcomes of the MIP for some test instances show the potential of the integrated approach and forms the base to investigate two heuristic approaches, which are better suitable for practical purposes. The latter is because of two reasons: (1) a heuristic approach reduces the time spend on calculating a solution significantly and may avoid that the hospital is required to invest in specific solver software, and (2) many heuristics can be designed in a way that they result in several good solutions instead of one optimal solution, which is preferred by the JBH. The heuristics provide pragmatic solutions to a real-world scheduling problem at the gynecology department that are acceptable for the gynecologists, the outpatient clinic management and the patients. The gynecologists and management at the JBH were involved in the entire process, and provided significant input for the developed model and the heuristics.

This paper is organized as follows. In Section 2 we clarify all case study details, and in Section 3 we provide the model definition and the heuristics. We end with a presentation of our results in Section 4 and a conclusion in Section 5. Note that although the MIP is presented for a specific gynecology department, the model is fairly general as many hospital departments have similar constraints on their specialist schedules, and the exact formulation of the constraints is readily tailored to other applications.

\section{Case study setting}

In this section we introduce the case study of the gynecology department of the JBH. The main goal for this research is to automatically create a schedule for the gynecologists, such that the outpatient clinic hours are balanced over all weeks, gynecologist preferences are better taken into account, and to significantly reduce the time that the scheduler has to spend on creating the schedules. To clarify this situation, we use an example of a fourweek schedule as used by the JBH, which is displayed in Table 1.

As can be seen in Table 1, there are many different task types in the schedule, for example 'on duty' (6 different task types), different specialized outpatient clinic sessions such as oncology, ultrasound, and general outpatient clinic sessions at different cooperating hospitals/separate locations. Each task type should be scheduled a certain number of times per week, not necessarily performed by the same gynecologist each time. Some gynecologists have specializations, which implies that not all task types may be performed by all gynecologists (see Table A.6 in the Appendix for all specializations).

Each weekday of the schedule consists of three shifts: morning, afternoon and evening. Each of these shifts takes about four hours, for which the exact working times (e.g., start at 8 a.m. or 8:30 a.m.) may be determined by the gynecologists. Thus, when a task type is assigned to a shift, this implies that this task is performed for about four hours by the gynecologist that is assigned to that task and shift. One exception to this rule are the 'on duty' task types on evenings; these gynecologists have to be available for consultation the entire night, but do not necessarily work the entire night (and often sleep at the hospital during their shift). Every day two gynecologists are assigned to on duty tasks: the first is assigned to the obstetrics 
Table 1

Example of a manual four-week schedule. Gyn 4 has been replaced by a different gynecologist during this research.
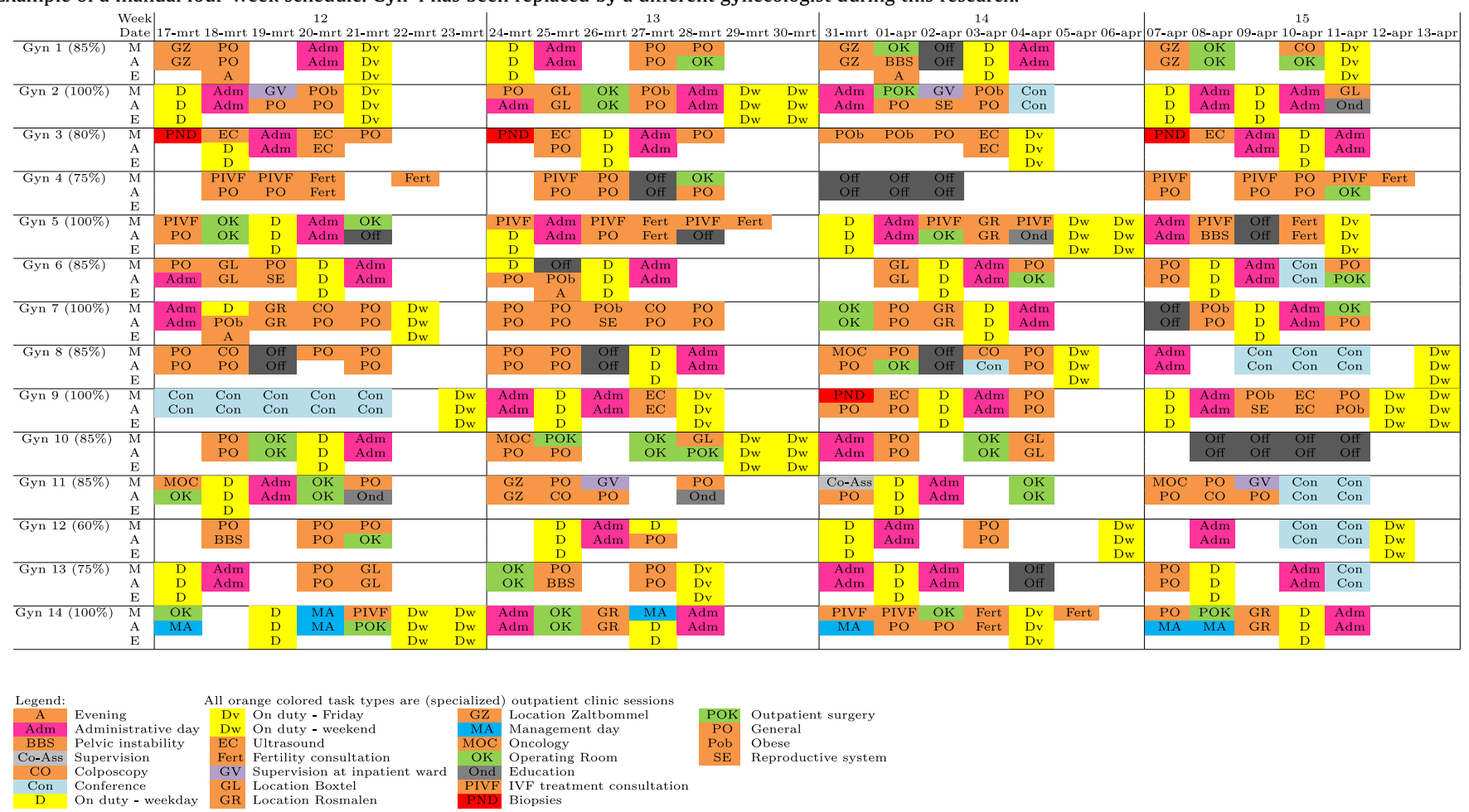

ward and the second to the other emergency patients and to assist the first gynecologist during times with high workload. After the often demanding on duty shifts on weekday evenings and weekends, a gynecologist is assigned to (the relatively low in workload) administrative shifts the next day.

Not all gynecologists work full-time at the JBH. One full-time contract should be equivalent to approximately $46 \mathrm{~h}$ per week. Several gynecologists work a fraction of a full-time contract, for example $85 \%$. These part-time gynecologists may be structurally unavailable on several days or day parts; gynecologist 5 for example never works on Wednesdays. Part-time gynecologists do have to work their fair (with respect to their contracted hours) share of weekend on duty shifts. One full-time gynecologist is allowed 7 weeks holidays and 10 conference days per year.

The scheduler has to assign gynecologists to shifts and tasks in such a way that the gynecologists work approximately the number of contracted hours. However, the scheduler always has to schedule the minimum number of tasks to ensure the accessibility of the clinic for patients, irrespectively of the contracted weekly hours of the gynecologists. Moreover, the actual time it takes to complete a task is not registered and there is no explicit check if working hour regulations are satisfied. At the moment, the JBH has enough gynecologists to cope with the workload at the hospital, so the gynecologists rarely have to work more than their contracted hours.

The on duty task types for weekends require six shifts from a gynecologist and for weekdays three (plus the two subsequent administration shifts), which significantly limits the scheduling options. There are seven task types that may only be performed by three or less gynecologists (for each task type a different subset). This implies that if two of them are on duty in the weekend, it is likely that these task types cannot be scheduled often enough in the subsequent week. Therefore, assigning task types to shifts first and later assign gynecologists, will most likely not lead to a feasible schedule. On the other hand, assigning gynecologists to shifts first and later assign the task types also significantly reduces the chances of obtaining a feasible schedule, as most task types have a limited subset of shifts and gynecologists to which they may be assigned. To overcome this problem, in this paper we simultaneously assign tasks and gynecologists to shifts.

At the JBH, one of the gynecologists is responsible for creating the schedules. As the gynecologist creates the schedules manually, this task is very time-consuming. Moreover, the gynecologists and the manager of the outpatient clinic are not satisfied with the quality of the schedules. To this end, in the next section we present our approach to automatically create better schedules for the department.

\section{Model}

In this section we first give an MIP model for the problem of creating suitable schedules for the gynecology department at the $\mathrm{JBH}$. Afterwards we introduce two heuristic approaches for the same problem. Because the MIP formulation is tailored for the gynecology department at the JBH, it may consist of constraints that are not applicable to other departments or hospitals. However, the model is fairly general, as many hospital departments have similar constraints on their specialist schedules, and the exact formulation of the constraints is readily tailored to other applications.

\subsection{MIP formulation}

The main decisions in creating a schedule for the gynecology department are to decide during which shifts each gynecologist has to work, and which task types they have to carry out during these shifts. To formalize the problem, in the following the sets, parameters and variables required for modeling this scheduling problem are introduced, and presented together with the hard and soft constraints and the objective of the MIP. The MIP formulation allows for incorporating many types of preferences and details with respect to prioritizing certain task types or shifts.

Let $S=\{1 m, 1 a, 1 e, 2 m, 2 a, 2 e, \ldots\}$ denote the set of shifts in the planning horizon. Here, the number indicates the day (e.g., ' 1 ' for the first day in the schedule, ' 2 ' for the second day, etc.) and the 
letters ' $m$ ', ' $a$ ' and ' $e$ ' denote the morning, afternoon and evening shift, respectively.

The tasks of the department can be grouped into $n^{T}$ different task types, which set is denoted by $T=\left\{1, \ldots, n^{T}\right\}$. The tasks have to be performed by a certain number of gynecologists working at the department, which are denoted by a set $G=\left\{1, \ldots, n^{G}\right\}$. The decision variables describing the schedule after the optimization are denoted by $x_{g s t}$, and are indicators that equal one if gynecologist $g \in G$ is scheduled for a task of type $t \in T$ in shift $s \in S$.

Each gynecologist can be assigned at most one task per shift, which is ensured by the constraint:

$$
\sum_{t \in T} x_{g s t} \leq 1 \quad \forall g \in G, s \in S .
$$

There may be task types that cannot be scheduled in all shifts, for example we cannot schedule a weekend on duty task to a weekday. Therefore, we define set $S N(t) \subset S$ as the set of shifts in which task type $t$ may not be scheduled. Additionally, gynecologists may not always be available due to holidays and other obligations. We define $S^{h}(g) \subset S$ as the set of shifts that gynecologist $g$ is unavailable due to holidays or other obligations. Furthermore, each gynecologist is specialized in different tasks, so we define $G(t) \subset G$ as the set of gynecologists qualified to perform task type $t$. We ensure that each task may only be assigned to qualified gynecologists and allowed shifts, by the following constraints:

$x_{\text {gst }}=0 \quad \forall g \notin G(t), s \in S, t \in T$,

$x_{g s t}=0 \quad \forall g \in G, s \in\left\{S N(t), S^{h}(g)\right\}, t \in T$.

For each task type a minimum and maximum frequency is specified, denoted by $l_{t}$ and $h_{t}$, respectively. These frequencies specify how many times this task should/may be scheduled each week. The task type 'POK' (outpatient surgery) has a different requirement: it should be scheduled at least once per week and exactly six times every four weeks. To enhance readability, we define subset $S W(j) \subset S$ to be all shifts of week $j, j \in J=$ $\left\{1,2, \ldots, n^{J}\right\}$, so $S W(1)=\{1 m, 1 a, 1 e, \ldots, 7 m, 7 a, 7 e\}$. Then, the following constraints set boundaries for the frequencies of each task type:

$$
\begin{array}{ll}
\sum_{g \in G} \sum_{s \in S W(j)} x_{g s t} \geq l_{t} & \forall j \in J, t \in T, \\
\sum_{g \in G} \sum_{s \in S W(j)} x_{g s t} \leq h_{t} & \forall j \in J, t \in T, \\
\sum_{g \in G} \sum_{s \in \bigcup_{i=0}^{3} S W(4 j-i)} x_{g s t}=6 & \forall 4 j \in J, t=\text { 'POK'. }
\end{array}
$$

The task types that refer to on duty play a special role at the department. We define $O=\{D, D v, D w\}$ as the set of task types that refer to on duty. In contrast to other task types, there should always be exactly two gynecologists per shift on duty. The following constraints ensure this:

$$
\sum_{g \in G} x_{g s t}=2 \quad \forall s \in S, t \in O .
$$

When a gynecologist is on duty for a weekday afternoon shift, she must also be on duty in the consecutive evening shift. For weekend days the constraint is different, as gynecologists may only be on duty an entire weekend. Let $S^{m}, S^{a}, S^{e}$ denote the subsets of $S$ containing all shifts corresponding to mornings, afternoons and evenings, respectively. Additionally, define $S^{w} \subset S$ as the set of weekend shifts. Let $A(s, n) \in S$ denote the $n$th shift after shift $s$, so for example $A(1 e, 2)=2 a$. Then, the following constraints ensure that on duty shifts on weekday afternoons are followed by an evening on duty shift, and one gynecologist should be on duty for all six shifts of a weekend:

$\begin{array}{ll}x_{g s t}=x_{g s^{\prime} t} & \forall g \in G, s \in S^{a} \backslash S^{w}, s^{\prime}=A(s, 1), t \in O, \\ x_{g s t}=x_{g s^{\prime} t} & \forall g \in G, s, s^{\prime} \in S^{w}, t \in O, \exists j \text { with } s, s^{\prime} \in S W(j) .\end{array}$

Because weekend on duty shifts are often demanding for gynecologists, after these shifts a gynecologist is assigned to a task of type 'adm' (administration) for one morning and afternoon shift. Let $S^{s e} \subset S^{w}$ denote the set of evening shifts on all Sundays in the schedule. The following constraints set the administration task type after a weekday and weekend with on duty:

$$
\begin{aligned}
x_{g s t}=x_{g s^{\prime} t^{\prime}} & \forall g \in G, s \in\left\{S^{e} \backslash S^{w}, S^{s e}\right\}, s^{\prime} \in\{A(s, 1), A(s, 2)\}, \\
& t \in O, t^{\prime}=\text { 'adm'. }
\end{aligned}
$$

Next to all hard constraints on the schedule, the gynecologists have expressed several preferences that would improve the schedule if they were satisfied. To this end, we introduce penalty variables that measure the deviation from the set target. The objective function of the MIP is a weighted sum of the penalty variables. In the following we define several soft constraints and penalty variables.

Besides a minimum and maximum frequency, each task type has a preferred minimum number of times per week it should be scheduled, denoted by $f_{t}$. The number of shifts that task type $t$ is scheduled less than $f_{t}$ in week $j$, is measured with penalty variable $b_{j t}$. Furthermore, at the JBH the task type 'Ond' (education, in which gynecologists for example educate interns) is preferably scheduled once every two weeks. We introduce penalty variable $c_{j}$ as the number of shifts that task type 'Ond' is scheduled less than once every two weeks. The following constraints ensure the preferred task type frequencies:

$$
\begin{array}{ll}
\sum_{g \in G} \sum_{s \in S W(j)} x_{g s t}+b_{j t} \geq f_{t} & \forall j \in J, t \in T, \\
\sum_{g \in G} \sum_{s \in S W(2 j)} x_{g s t}+c_{j} \geq 1 & \forall 2 j \in J, t=\text { 'Ond'. }
\end{array}
$$

Gynecologists prefer not to have idle time during their workday, e.g., if they are scheduled for a morning and evening shift on a certain day, they prefer to work the afternoon shift too. Recall that the subset $S^{m}$ consists of all morning shifts. We introduce penalty variable $d_{g s}$, which equals one if gynecologist $g$ has idle time after shift $s$, and the following soft constraint to favor non-idle workdays:

$$
\begin{aligned}
& \sum_{t \in T} x_{g s t}-x_{g s^{\prime} t}+x_{g s^{\prime \prime} t}-d_{g s} \leq 1 \quad \forall g \in G, \\
& s \in S^{m}, s^{\prime}=A(s, 1), s^{\prime \prime}=A(s, 2) .
\end{aligned}
$$

Furthermore, if a gynecologist is on duty in a certain shift on a weekday, it is preferred that she also works the other on duty shifts on the same day. This only holds for weekday shifts, as the weekend on duties are constrained by (7) and (8). We introduce penalty variable $i_{g s}$ that equals one if gynecologist $g$ is assigned at least one but less than three on duty shifts on the day containing shift $s$. The following constraints allow to favor schedules in which one on duty type is assigned to the same gynecologist in all shifts of a single day:

$$
\begin{array}{ll}
i_{g s} \geq x_{g s t}-x_{g s^{\prime} t} & \forall g \in G, s \in\left\{S^{m}, S^{a}\right\} \backslash S^{w}, s^{\prime}=A(s, 1), t \in O . \\
i_{g s} \geq x_{g s^{\prime} t}-x_{g s t} & \forall g \in G, s \in\left\{S^{m}, S^{a}\right\} \backslash S^{w}, s^{\prime}=A(s, 1), t \in O .
\end{array}
$$

A good schedule takes each gynecologist's contracted working hours and holidays into account, and assigns approximately the fair 
number of shifts for each gynecologist with respect to their contracted hours. We define parameter $r_{g}$ as the ratio of gynecologist $g$ 's contracted working hours to one fte (full-time equivalent), for example an fte ratio of 0.85 implies $85 \%$ of 46 contracted hours per week. With this parameter we construct parameter $r_{g j}^{s}$, which is the number of shifts gynecologist $g$ should work in week $j$ according to the contracted working hours. $r_{g j}^{s}$ is determined by dividing the preferred number of shifts per week, $\sum_{t} f_{t}$, over the gynecologists according to the fte ratios. For example, in weeks without holidays the preferred total number of shifts equals 119 and all gynecologists together work for 12.15 fte. Therefore, a gynecologist who works 1 fte should be assigned to $119 / 12.15=$ 9.79 shifts, and a gynecologist working 0.85 fte should work 0.85 . $9.79=8.32$ shifts per week. In parameter $r_{g j}^{s}$ holiday week shifts are taken into account, to prevent that a gynecologist is assigned to too many shifts surrounding her holiday weeks. It is likely that $r_{g j}^{s}$ contains mostly fractional values, implying that the number of shifts cannot be assigned exactly according to the fte ratios. We quantify 'fairness' in the schedule by the maximum deviation from a desired value. Therefore, we define penalty variables $k_{g j}$ and $\bar{k}_{g j}$ as the total number of shifts gynecologist $g$ is under- and overscheduled in week $j$, respectively. The following soft constraints set these penalty variables:

$$
\begin{array}{ll}
\sum_{s \in S W(j)} \sum_{t \in T} x_{g s t}+k_{g j} \geq\left\lfloor r_{g j}^{s}\right\rfloor & \forall g \in G, j \in J, \\
\sum_{s \in S W(j)} \sum_{t \in T} x_{g s t}-\bar{k}_{g j} \leq\left\lceil r_{g j}^{s}\right\rceil & g \in G, j \in J .
\end{array}
$$

Here, $\lceil r\rceil$ and $\lfloor r\rfloor$ denote $r$ rounded up and down to the nearest integer, respectively. Constraints (15) and (16) would not prevent that one of the gynecologists has the maximum deviation (number of shifts over or under $r_{g j}^{s}$ ) every week of the schedule, which would still imply an unfair schedule. Therefore, we additionally introduce two similar constraints for the entire scheduling horizon. Let penalty variables $p_{g}$ and $\bar{p}_{g}$ denote the number of shifts gynecologist $g$ is under- and over-scheduled over the entire scheduling horizon, respectively. The following constraints set these penalty values:

$$
\begin{array}{ll}
\sum_{s \in S} \sum_{t \in T} x_{g s t}+p_{g} \geq\left\lfloor\sum_{j \in J} r_{g j}^{s}\right\rfloor & \forall g \in G, \\
\sum_{s \in S} \sum_{t \in T} x_{g s t}-\bar{p}_{g} \leq\left\lceil\sum_{j \in J} r_{g j}^{s}\right] & \forall g \in G .
\end{array}
$$

At the gynecology department there are many different task types to be performed. The number of times each qualified gynecologist performs each task type should be fair with respect to their contracted working hours, and should be balanced for each gynecologist over the entire scheduling horizon. Again, we quantify fairness in the schedule by the maximum deviation from a desired value. Penalty variable $q_{t}$ measures for each task type $t$ the maximum difference between the number of tasks of this type that are performed by gynecologist $g$ and the number of times $g$ is expected to perform task type $t$ according to the fte ratios $r_{g}$. For example, there are two gynecologists allowed to perform task type 'PIVF' (namely 5 and 14), who together work for 1.85 fte. PIVF is preferably performed three times per week, so twelve times in a four-week schedule. This implies that gynecologist 5 should be assigned to PIVF $12 / 1.85=6.49$ times and gynecologist 14 to $6.49 \cdot 0.85=5.51$ PIVF shifts. Furthermore, constraint (20) ensures that the number of times each task is performed, is balanced over the weeks for each gynecologist, where penalty variable $u_{g t}$ denotes the maximum number of times gynecologist $g$ performs a task of type $t$ in one week.

$$
\begin{aligned}
\sum_{s \in S} x_{g s t} \leq q_{t} r_{g} & \forall g \in G, t \in T, \\
\sum_{s \in S W(j)} x_{g s t} \leq u_{g t} & \forall g \in G, j \in J, t=T \backslash\{O, \operatorname{adm}\} .
\end{aligned}
$$

Not all soft constraints are equally important to the gynecologists and hospital management. Therefore, for each penalty variable we introduce a penalty parameter that reflects the weight of each penalty variable in the objective function. Each penalty parameter is specified in the same detail as the accompanying variable is defined, to allow the JBH to set specific preferences if necessary. For example, the preferences of a certain gynecologist, task type, shift, week, or any combination of the indices may be prioritized in the optimization.

All penalty variables and parameters together determine the weighted objective function of this MIP:

$$
\begin{aligned}
& \text { Minimize } \sum_{j \in J, t \in T} w b_{j t} b_{j t}+\sum_{j \in J} w c_{j} c_{j}+\sum_{g \in G, s \in S^{m}} w d_{g s} d_{g s} \\
& +\sum_{g \in G, s \in S^{m} \backslash S^{w}} w i_{g s} i_{g s} \\
& +\sum_{g \in G, j \in J} w k_{g j}\left(k_{g j}+\bar{k}_{g j}\right)+\sum_{g \in G} w p_{g}\left(p_{g}+\bar{p}_{g}\right)+\sum_{t \in T} w q_{t} q_{t} \\
& +\sum_{g \in G, t \in T \backslash\{0, \text { adm. }\}} w u_{g t} u_{g t} .
\end{aligned}
$$

All variables in the MIP are non-negative, with variables $x_{\text {gst }}$ restricted to binary values and $c_{j}$ to the interval $[0,1]$. Although this objective function is tailored to the JBH application, the modeling approach allows for tailoring diverse types of constraints to other applications.

Solving the program for realistic scheduling horizons requires substantial running time (at least over $12 \mathrm{~h}$ ) and specific software that is generally not available in hospitals. Note that the first disadvantage may be overcome by solving the given MIP formulation using a column generation approach. Generally, implementing such approaches requires substantial time to both reformulate the MIP and find a suitable branching strategy, and still requires specific software. This was the reason we investigated two heuristic approaches to approximate the MIP solution, which are presented in the next subsection.

\subsection{Heuristic approaches}

By simultaneously assigning tasks and gynecologists to shifts, we increase the solution space compared to sequential assignments. For practical applications, quickly generating a few good schedules is often preferred over obtaining the optimal schedule in longer computation time $[11,16]$. To this end, and because heuristics may be implemented in generally available software, we investigate several local search heuristics, as in many practical applications they have shown to reach high quality solutions and visit many good solutions during their run. In the following, we first clarify how initial solutions for the heuristics are generated, then provide several neighborhood search procedures, and finally describe a multi-start local search and a simulated annealing heuristic. The pseudo-code of the heuristics is provided in Algorithms 1 and 2 .

To apply local search procedures, an initial schedule that complies with all hard constraints has to be generated. The most difficult task types to schedule are the series of on duty shifts and administrative tasks. Therefore, we generate the initial schedule 

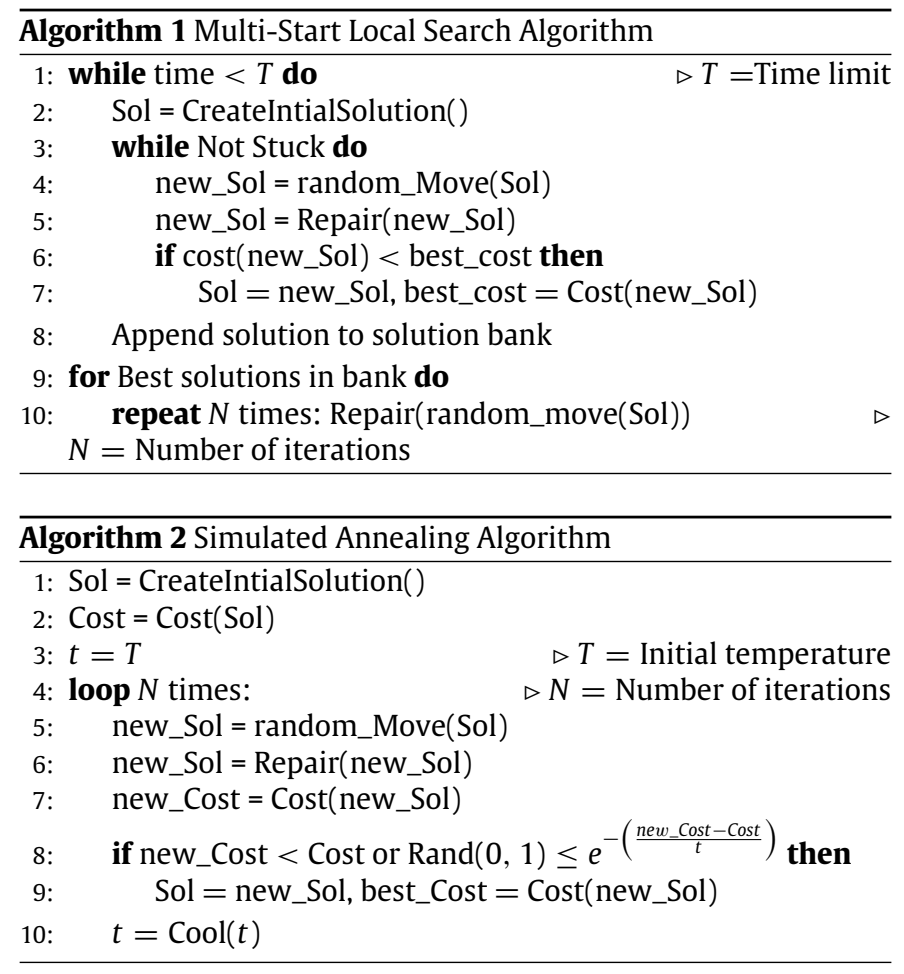

by first assigning these tasks in a 'weighted random' fashion; for each series of shifts the gynecologists who can perform many other tasks in this series are less likely to be assigned to on duty. To this end, we weight the probabilities according to the number of possible task allocations lost by scheduling a gynecologist to a particular set of shifts, which is determined by: (1) the number of different task types the gynecologist is qualified to perform, and (2) the number of shifts the gynecologist is available. Next, we schedule all task types with a minimum scheduling requirement and assign them to gynecologists and shifts following a similar least regret paradigm:

- we choose a task randomly, favoring task types by settings weights that have a relatively high number left to schedule and have less possible shifts to be assigned to,

- and we choose a gynecologist/shift combination from the subset of allowed combinations randomly, favoring combinations via weights that allow less possible task assignments.

Finally, we schedule the additional outpatient surgery task types randomly, again using weights. These task types have a monthly instead of weekly target and we again favor the gynecologist/shift combinations that allow less other task assignments. Note that the scheduling procedure may get stuck without all necessary number of tasks scheduled due to the random choices at each iteration. If at any point there is no allowed assignment for a task, we restart the complete procedure. This procedure appears to generate feasible initial solutions quickly, often without restarts.

The two heuristics described below use a neighborhood search procedure in each iteration. The neighborhood operators investigated are: (1) allocate task, (2) delete task, (3) swap tasks, and (4) reassign on duty shifts. As the initial schedule contains only the minimum required number of each task type, there are still tasks that may be added after initialization. Tasks may only be deleted if their minimum frequency is not violated. Furthermore, tasks may be swapped, i.e., the gynecologist/shift combination of the two tasks are interchanged, if: (1) the tasks are of different types, (2) both are not on duty or administrative days, (3) they are scheduled in the same week, and (4) the new gynecologist/shift combination is allowed. Reassigning on duty shifts requires a series of swaps and several repair operations, as these task types involve a series of consecutive shifts. When reassigning weekend on duty shifts, the administrative task shifts of the following Monday are also reassigned. After the reassignment of these series, a repair procedure is initiated that tries to either reschedule or delete all tasks that had to be removed from the schedule before the reassignment. If the repair procedure is not successful, or at any point during this neighborhood operator the resulting schedule would become infeasible, all alterations to the schedule during this iteration are reversed. All developed approaches work only with feasible solutions, to ensure a user can terminate the heuristic at any time and still obtains a feasible schedule.

The neighborhood search procedures are integrated in a multistart local search (LS) heuristic and a simulated annealing (SA) algorithm. During each iteration in both heuristics one of the neighborhood search procedures is selected randomly, and after its execution a repair procedure is called ensuring that the outpatient surgery is still performed six times each month. The LS heuristic explores the neighborhood of solutions randomly, only accepting new schedules with better objective value, until a certain number of iterations without improvements to the schedule is reached. This solution is then stored, and the heuristic is restarted from a new initial schedule. After a number of restarts depending on the length of the horizon, the heuristic is reapplied to the best found solutions so far for a limited number of iterations. For the SA heuristic, we use the standard acceptance rule and an exponential cooling scheme, see for example [17] for more information.

\section{Results}

This section contains computational and practical results of the MIP model and heuristic approaches. In Section 4.1 we specify the investigated scheduling horizons, the number of gynecologists and tasks, and all input for both heuristics. In Section 4.2 we provide insights in both the solution speed and quality of the MIP and the heuristics for different scheduling horizons and scenarios based on data from the JBH.

\subsection{Case study specific input}

For the MIP and both heuristics we investigate planning horizons of four, eight, and twelve weeks; $n^{J} \in\{4,8,12\}$. As the JBH currently employs 14 gynecologists, and the department distinguishes 25 different task types, we use these numbers also in our scenarios. The gynecologists and hospital management together determined weight parameters for the necessary penalty variables by manually evaluating several schedules obtained using the MIP. After four iterations on several of the parameter settings, the gynecologists and hospital management were satisfied with the resulting schedule. In the chosen parameter settings, the task type 'general outpatient clinic' (there are also several specialized outpatient clinic task types) has the highest priority, because the JBH experienced difficulties in scheduling patients in the medically preferred weeks of their pregnancy. Another example task type with high weight is one of the specialized outpatient clinics, in which the gynecologists cooperate with other specialties. All input for this case study is provided in Appendix.

In a first series of tests we consider an instance in which all gynecologists are fully available. As the JBH indicated that holiday periods are generally complicated to schedule, as a second series of tests we consider instances with varying availabilities. Creating good schedules is especially hard during periods in which many gynecologists are unavailable, because each gynecologist has her 
Table 2

Hard constraint violations in the manual four-week schedule.

\begin{tabular}{lc}
\hline Hard constraint & No. violations \\
\hline Allowed times for tasks & 29 \\
Allowed gynecologist assignment & 8 \\
Below minimum frequency & 14 \\
Above maximum frequency & 58 \\
POK 6 times in four weeks & 0 \\
Always someone on duty & 0 \\
If weekday afternoon then evening on duty & 0 \\
On duty entire weekend & 3 \\
Adm shift after weekday and weekend on duty & 3 \\
Fixed days off & 7 \\
\hline Total & $\mathbf{1 2 2}$ \\
\hline
\end{tabular}

own specializations and there are specialized tasks that may only be performed by one or few gynecologist(s). This may result in unsolvable instances if all gynecologists for such a task have holidays in the same weeks. However, for holiday weeks there are no penalty scores for over- or under-scheduling a gynecologist as $r_{g s}^{s}=0$ for those weeks. Therefore, it does not hold generally that holiday weeks always result in schedules with higher objective values.

For practical purposes, we limit the solution time of the MIP to 12,48 and $72 \mathrm{~h}$ for the four-, eight- and twelve-week horizons investigated. We choose the parameters of the heuristics such that they also terminate within one hour. The MIP was programmed in AIMMS 4.36 and solved using Gurobi 7.0. Both heuristics were programmed in Python 3.6. For the LS heuristic, we limit the first phase to $4 / 5 \mathrm{~h}$. Preliminary experiments indicated that limiting the number of iterations without an improved solution to 500 results in the best balance between exploring new solutions and prematurely judging solutions as 'stuck' in local optima. In the stage of reapplying the LS heuristic to the best solution initially found by the algorithm, we limit the running time to $1 / 5$ of the one hour total solution time. With the limitation on the running time, this implies that for the four week horizon the heuristic returns three solutions, and for the eight and twelve week horizons two solutions are returned. In the SA algorithm, based on the results of initial experiments, we set the initial temperature at 300 and use a cooling coefficient of 0.93 , lowering the temperature 100 times each run. This results in a final temperature of about 0.21 . All numerical results are obtained using an Intel Quad Core i7-2630Q CPU $2.0 \mathrm{GHz}$ with $4 \mathrm{~GB}$ RAM.

\subsection{Computational results of the methods}

The developed methods are only useful in practice if they can deliver good schedules for all practically relevant scenarios within reasonable time. In this subsection, we first investigate the performance of the MIP and both heuristics for a basic scenario without holidays, which are the easiest to solve in practice.

One of the most valuable tests for the JBH is to compare the computerized schedules to the schedules currently used by the $\mathrm{JBH}$. To be able to make this comparison, we determine the penalty variables for a manually created schedule. However, finding a four week period without any gynecologists on holidays or conferences appeared impossible, and we took a four week schedule from the JBH with: one fte unavailable in week one, $0.75 \mathrm{fte}$ in week three, and $1.7 \mathrm{fte}$ in week four. This schedule did not satisfy all hard constraints, see Table 2. When we ignore the hard constraint violations, the objective value of this manual schedule is 3063 , which should be regarded as an approximation.

Table 3 displays the contribution of each soft constraint to the objective value for all base scenarios and the manual schedule, where $b$ denotes $\sum w b_{j t} b_{j t}$ and likewise for all penalty variables of the objective function (21). The break-down of the objective function for the manual schedule confirms the believe of the gynecologists and manager at the JBH; the frequency of each task type per week is severely unbalanced (parameter $q$ ), there are several days on which not all on duty shifts are assigned to the same gynecologist $(i)$, and the number of assigned shifts per gynecologist is relatively unfair ( $k$ and $p$ ). Both the MIP model and the heuristics obtain better schedules in less time compared to the manually obtained schedule, improving the compliance to all soft constraints except $u$, the maximum number of times a gynecologist performs a task in one week. From these results it appears that the scheduler has prioritized $u$ in the manual schedule, as this contribution to the objective value is significantly higher in the MIP and heuristic schedules. However, after a visual inspection of the obtained schedules by the scheduler, an other gynecologist and the manager, all approved the proposed schedules and decided to keep the penalty parameter values as displayed in Appendix.

The gap displayed in Table 3 is calculated using the MIP lower bound, and is still quite high for both heuristics. However, as the heuristics only ran one hour on a fraction of the computer's capacity (AIMMS uses all cores with parallel computing), these results are promising. Based on these experiments, the LS heuristic seems to outperform the SA heuristic.

To investigate the performance of our methods in more practically relevant settings, in the following we investigate our methods for different scenarios including summer holiday periods. At the $\mathrm{JBH}$, the summer holiday period consists of six weeks, in which each gynecologist with children may take either the first three weeks (group $G^{f}$ ) or the last three weeks $\left(G^{l}\right)$ off. Approximately $1 / 3$ of the gynecologists does not have children $\left(G^{n}\right)$, and take their three holiday weeks outside this holiday period. At least one of the gynecologists who may perform a specialized task should be available each week.

To test our methodology in holiday periods, we created three test cases for a summer holiday period. The three test cases 1,2 , and 3 differ in the distribution of gynecologists over the groups $G^{f}, G^{l}$ and $G^{n}$, and get harder with increasing number. Case 1 is a 'best case scenario', in which all gynecologists allowed to perform highly specialized task types are in $G^{n}$. For all other task types, we balance the number of gynecologists that may perform this task type evenly over the groups $G^{f}$ and $G^{l}$. Case 2 is more difficult to schedule as the gynecologists in $G^{n}$ are not qualified for the highly specialized task types, but the remaining gynecologists are still spread evenly over other groups according to their specializations. Case 3 is the hardest to schedule, as all gynecologists with the lowest number of contracted fte hours are placed in $G^{n}$. The other gynecologists are again spread evenly over the other groups. The scheduling period was set to eight weeks, of which the first six were holiday weeks.

For all three cases, all methods still resulted in feasible schedules, satisfying all hard constraints. The optimized schedules in holiday weeks satisfy, as expected, fewer soft constraints, see Table 4. Schedules for periods with and without holidays are difficult to compare. However, the obtained schedules for Case 1 and 2 are still significantly better than the manual (four week) schedule without holidays. This indicates the practical relevance of the schedules in holiday periods for the JBH. Additionally, the difference between the objective values for the hardest Case 3 and best-case scenario Case 1 is relatively small, which is promising for the quality of the schedule for even harder instances. The performance of the heuristics is significantly better for holiday cases than for the base scenario; the LS heuristic has obtained the MIP lower bound for case 3 after only one hour running time. A possible explanation for the good results in the holiday cases, is that the number of variables is reduced significantly due to the holiday unavailability of the gynecologists. Again, the LS heuristic outperforms the SA heuristic for the holiday cases. 
Table 3

Analytical results for different horizons, '4 - MIP' denotes the MIP schedule for a four-week horizon, and $b$ denotes $\sum w b_{j t} b_{j t}$ and likewise for all penalty variables of the objective function.

\begin{tabular}{lrllrrrrrrrr}
\hline Schedule & Time $(\mathrm{s})$ & Gap & Obj. val. & \multicolumn{1}{l}{$b$} & \multicolumn{1}{c}{$c$} & $d$ & $i$ & $k$ & \multicolumn{1}{l}{$p$} & \multicolumn{1}{c}{$q$} \\
\hline 4 - Manual & 172800 & & 3063 & 105 & 0 & 0 & 500 & 270 & 318 & 1446 & 424 \\
4 - MIP & 86402 & $6,83 \%$ & 1018 & 0 & 0 & 0 & 0 & 0 & 0 & 162 & 856 \\
4 - SA & 3600 & $46 \%$ & 1752 & 207 & 0 & 0 & 0 & 96 & 126 & 178 & 1145 \\
4 - LS & 3557 & $34 \%$ & 1437 & 196 & 7 & 0 & 0 & 72 & 81 & 192 & 889 \\
\hline 8 - MIP & 172803 & $11,30 \%$ & 1747 & 14 & 0 & 0 & 0 & 0 & 0 & 190 & 1543 \\
8 - SA & 3600 & $49 \%$ & 3019 & 385 & 0 & 0 & 0 & 174 & 189 & 201 & 2061 \\
8 - LS & 3947 & $42 \%$ & 2678 & 326 & 7 & 0 & 0 & 195 & 213 & 224 & 1713 \\
\hline 12 - MIP & 259200 & $6,21 \%$ & 2387 & 5 & 0 & 0 & 20 & 0 & 0 & 202 & 2160 \\
12 - SA & 3600 & $48 \%$ & 4320 & 581 & 7 & 0 & 0 & 294 & 219 & 232 & 2987 \\
12 - LS & 4349 & $35 \%$ & 3446 & 608 & 14 & 0 & 0 & 252 & 168 & 238 & 2166 \\
\hline
\end{tabular}

Table 4

Results for an eight week horizon for three holiday cases.

\begin{tabular}{lrllrrrrrrrr}
\hline Schedule & Time (s) & Gap & Obj. val. & \multicolumn{1}{l}{$b$} & \multicolumn{1}{c}{$c$} & $d$ & $i$ & $k$ & $p$ & $q$ & $u$ \\
\hline Base - MIP & 172803 & $11.30 \%$ & 1747 & 14 & 0 & 0 & 0 & 0 & 0 & 190 & 1543 \\
Base - SA & 3600 & $49 \%$ & 3019 & 385 & 0 & 0 & 0 & 174 & 189 & 201 & 2061 \\
Base - LS & 3600 & $42 \%$ & 2678 & 326 & 7 & 0 & 0 & 195 & 213 & 224 & 1713 \\
\hline Case 1 - MIP & 43200 & $5.49 \%$ & 2503 & 882 & 28 & 0 & 0 & 144 & 195 & 162 & 1092 \\
Case 1 - SA & 3600 & $18 \%$ & 2870 & 350 & 0 & 0 & 0 & 195 & 243 & 216 & 1866 \\
Case 1 - LS & 3935 & $4 \%$ & 2457 & 290 & 7 & 0 & 0 & 141 & 198 & 240 & 1581 \\
\hline Case 2 - MIP & 43203 & $6.07 \%$ & 2579 & 818 & 21 & 0 & 0 & 204 & 252 & 174 & 1110 \\
Case 2 - SA & 3600 & $19 \%$ & 3001 & 343 & 7 & 1 & 0 & 132 & 231 & 210 & 2077 \\
Case 2 - LS & 3941 & $4 \%$ & 2533 & 332 & 7 & 0 & 0 & 144 & 159 & 210 & 1681 \\
\hline Case 3 - MIP & 43200 & $4,76 \%$ & 2920 & 951 & 21 & 0 & 0 & 264 & 321 & 178 & 1185 \\
Case 3 - SA & 3600 & $8 \%$ & 3008 & 364 & 0 & 0 & 0 & 231 & 297 & 220 & 1896 \\
Case 3 - LS & 3914 & $0 \%$ & 2768 & 331 & 7 & 0 & 0 & 174 & 171 & 226 & 1859 \\
\hline
\end{tabular}

Currently, the JBH uses a six-week scheduling horizon. In this research, we used multiples of four weeks because of constraint (5). The JBH indicated that they want to extend the scheduling horizon, but that this was an impossible task to ask from the current scheduler. Our results indicate that good schedules can still be obtained within a reasonable amount of time for longer scheduling horizons. The difference between an eight- and a twelve-week schedule is determined mainly by parameters $b_{j t}$ and $u_{g t}$. From our results it is not clear which horizon length is best for the JBH, but our methods obtain good schedules swiftly for both lengths.

So far, the performance of the investigated methods is assessed through the objective value of the best found schedule. However, in practice it is hard to decide in a fully automated fashion if a schedule is better than another schedule. Despite the time invested in prioritizing the soft constraints, among two schedules with similar objective values, gynecologists might have a clear preference, and gynecologists might be indifferent between two schedules with distinct objective values. Although the improvements in the objective value of the schedules are significant, the main practical gain of this research is the significant reduction of manual scheduling time. Furthermore, the benefits of heuristics over an exact approach are that heuristics are particularly useful for providing multiple distinct, good schedules swiftly, so the scheduler has a good starting point for some manual fine-tuning. Both the LS and SA heuristics are therefore very useful in practice, and our numerical experiments show that the performance of both heuristics is good in practical situations. Additionally, creating schedules automatically implies that one gynecologist can be relieved from many hours of scheduling, and the fact that the resulting schedule has a better objective value than the manual schedule is promising for acceptance of the method in practice.

\section{Conclusion}

In this paper we presented solution approaches to assigning tasks and gynecologists to shifts for the gynecology department of the JBH. The obtained schedules satisfy all hard constraints and significantly more soft constraints compared to a four-week manual schedule used by the JBH less time. Even in supposedly hard holiday scenarios, our methods obtained good schedules in a fraction of the time usually spend on creating the schedules. The MIP results in good schedules, often reaching an optimality gap less than 7\% within one hour, but if the JBH does not want to invest in specific software, our heuristics have proven to rapidly result in several good schedules too.

The new contribution of this paper is that we simultaneously assign recurring tasks and gynecologists to available shifts, to incorporate specializations of the gynecologists that are not strictly increasing in hierarchy levels while each gynecologist may have different working hours. Furthermore, if the tasks were assigned first in a sequential assignment procedure, this significantly restricts the assignments of the gynecologists and vice versa, which may reduce the quality of the obtained schedule.

For testing the approach, we used the weights specified by the gynecologists and the department management of the JBH. In the obtained schedules, often the same tasks were under-scheduled. Therefore, in further research we may adjust the penalties parameters to also reflect the relative difficulty in satisfying the constraints.

The methods presented in this paper were all limited in running time to be of more practical relevance. For larger scheduling horizons, speeding up the solving process (for example by using a column generation approach) would become an interesting topic for further research. However, as stated before, the JBH favors several good solutions over one optimal solution, and prefers not to install specific MIP-solving software.

The two simple heuristics presented in this paper were pragmatic in solving the scheduling problem. A further extension to the current research would be to allow the neighborhood search procedures to temporarily visit infeasible solutions, to explore the solution space faster and avoid returning local optima. Additionally, as weekend on duty shifts should be divided fairly among 
Table A.5

$r_{g}$ for each gynecologist.

\begin{tabular}{ll}
\hline$r_{g}$ & Subset of $G$ \\
\hline 1 & $\{2,5,6,7,9\}$ \\
0.85 & $\{1,8,10,11,14\}$ \\
0.8 & $\{3\}$ \\
0.75 & $\{4,13\}$ \\
0.6 & $\{12\}$ \\
\hline
\end{tabular}

Table A.6

$G(t)$ for each task.

\begin{tabular}{llll}
\hline Task type $t$ & $G(t)$ & Task type $t$ & $G(t)$ \\
\hline A & $\{1,6-10\}$ & GR & $\{5,7,14\}$ \\
Adm & $G$ & GZ & $\{1,11\}$ \\
BBS & $\{1,5,12,13\}$ & MA & $\{14\}$ \\
Co-Ass & $G$ & MOC & $\{8,10,11\}$ \\
CO & $\{1,7,8,10,11\}$ & OK & $G \backslash\{3\}$ \\
Con & $G$ & Ond & $\{7\}$ \\
D & $G$ & PIVF & $\{5,14\}$ \\
Dv & $G$ & PND & $\{3,4,9\}$ \\
Dw & $G$ & POK & $\{2,6,10,12,14\}$ \\
EC & $\{3,4,9\}$ & PO & $G$ \\
Fert & $\{5,14\}$ & POb & $\{2,3,4,6,7,9\}$ \\
GV & $G$ & SE & $\{1\}$ \\
GL & $\{2,6,10,12,13\}$ & & \\
\hline
\end{tabular}

the gynecologists over a year, incorporating information from the previous results from the MIP (a running horizon approach) would be an interesting extension to the current research.

Concluding, the exact and heuristic approaches result in better schedules obtained in significantly less time, also for realistic instances. When we presented the results at the JBH, the department was enthused about the improvement potential, and is considering to use the heuristic approaches in practice.

\section{Acknowledgments}

The authors are grateful for the input from all involved staff members and gynecologists of the JBH, and for the great help of Gerhard Post (University of Twente). Additionally, we gratefully acknowledge the anonymous referees and editor, who have helped to improve this paper significantly.

\section{Appendix. Parameters for the case study}

In this section we provide all sets and parameters for the JBH case study. Recall that $G=\{1, \ldots, 14\}$ and $T=\{1, \ldots, 25\}$. The fte ratio of each gynecologist, $r_{g}$, is given in Table A.5.

The gynecologists with $r_{g}<1$ may specify weekday shifts in which they are not available: gynecologists 1,8 , and 13 are not available on all Wednesday shifts; gynecologist 3 is not available on the afternoon and evening shifts on Mondays and Fridays; gynecologist 4 is not available on the morning and afternoon shift on Fridays; and gynecologist 12 is not available on all Monday and Thursday shifts.

The set of gynecologists that is allowed to perform task $t, G(t)$, is given in Table A.6. The set of on duty tasks is given by $0=$ $\{\mathrm{D}, \mathrm{Dv}, \mathrm{Dw}\}$.

We assume that the penalty parameters are the same for each week in the schedule. The penalty parameters that do not depend on any index are given in Table A.7. The task-specific (penalty) parameters are given in Table A.8. Note that the 'Con' tasks (visiting a conference) are requested by the gynecologist in specific shifts and are not part of the optimization.

Recall that $S N(t)$ denotes the set of shifts when task $t$ cannot be performed, and $S^{z}$ for $z \in\{w, m, a, e\}$ denotes the set of weekend, morning, afternoon and evening shifts, respectively. For the ease
Table A.7

Penalty parameters that do not depend on an index.

\begin{tabular}{llllll}
\hline Parameter & $w c$ & $w d$ & $w i$ & $w k$ & $w p$ \\
\hline Value & 7 & 1 & 20 & 3 & 3 \\
\hline
\end{tabular}

Table A.8

Task-specific (penalty) parameters.

\begin{tabular}{lrrrrrr}
\hline & $f$ & $l$ & \multicolumn{1}{c}{$h$} & $w b$ & $w u$ & $w q$ \\
\hline A & 1 & 0 & 1 & 9 & 2 & 4 \\
Adm & 20 & 20 & 20 & 0 & 0 & 0 \\
BBS & 1 & 0 & 1 & 6 & 2 & 5 \\
Co-Ass & 1 & 0 & 1 & 8 & 2 & 5 \\
CO & 2 & 2 & 2 & 0 & 2 & 8 \\
D & 24 & 24 & 24 & 0 & 2 & 20 \\
Dv & 6 & 6 & 6 & 0 & 0 & 20 \\
Dw & 12 & 12 & 12 & 0 & 0 & 20 \\
EC & 3 & 0 & 3 & 6 & 2 & 5 \\
Fert & 2 & 0 & 2 & 9 & 2 & 5 \\
GV & 1 & 0 & 1 & 6 & 2 & 5 \\
GL & 4 & 0 & 4 & 7 & 2 & 5 \\
GR & 3 & 0 & 3 & 8 & 2 & 5 \\
GZ & 2 & 0 & 2 & 7 & 2 & 5 \\
MA & 2 & 0 & 2 & 7 & 2 & 5 \\
MOC & 1 & 0 & 1 & 10 & 2 & 5 \\
OK & 10 & 10 & 10 & 0 & 2 & 8 \\
Ond & 1 & 0 & 1 & 0 & 0 & 5 \\
PIVF & 3 & 0 & 3 & 5 & 2 & 5 \\
PND & 1 & 0 & 1 & 6 & 2 & 5 \\
POK & 1 & 1 & 6 & 0 & 2 & 8 \\
PO & 10 & 7 & 13 & 10 & 2 & 5 \\
POb & 3 & 0 & 3 & 6 & 2 & 5 \\
SE & 1 & 0 & 1 & 8 & 2 & 5 \\
\hline & & 0 & & & & \\
\hline
\end{tabular}

Table A.9

$S N(t)$ for each task $t$.

\begin{tabular}{ll}
\hline Task type $t$ & $S N(t)$ \\
\hline A & $S \backslash\left\{S^{T u} \cap S^{e}\right\}$ \\
Adm & $\left\{S^{w}, S^{e}\right\}$ \\
BBS & $S \backslash\left\{S^{T u} \cap S^{a}\right\}$ \\
CO & $\left\{S^{e}, S \backslash\left\{S^{T u}, S^{T h} \cap S^{m}\right\}\right\}$ \\
Co-Ass & $S \backslash\left\{S^{F} \cap S^{a}\right\}$ \\
D & $\left\{S^{w}, S^{F}\right\}$ \\
Dv & $S \backslash S^{F}$ \\
Dw & $S \backslash S^{w}$ \\
EC & $S \backslash\left\{S^{T h} \cap S^{m}\right\}$ \\
Fert & $\left\{S^{e}, S \backslash S^{T u}\right\}$ \\
GL & $\left\{S^{e}, S^{M} S^{T h} S^{w}\right\}$ \\
GR & $\left\{S^{e}, S \backslash\left\{S^{W}, S^{T h}\right\}\right\}$ \\
GV & $S \backslash\left\{S^{W} \cap S^{m}\right\}$ \\
GZ & $\left\{S^{e}, S \backslash S^{M}\right\}$ \\
MA & $\left\{S^{w}, S^{e}\right\}$ \\
MOC & $S \backslash\left\{S^{M} \cap S^{m}\right\}$ \\
OK & $\left\{S^{w}, S^{e}\right\}$ \\
Ond & $\left\{S^{w}, S^{e}\right\}$ \\
PIVF & $\left\{S^{W}, S^{F}, S^{w}, S^{a}, S^{e}\right\}$ \\
PND & $S \backslash\left\{S^{M} \cap S^{m}\right\}$ \\
PO & $\left\{S^{w}, S^{e}\right\}$ \\
POb & $\left\{S^{w}, S^{e}\right\}$ \\
POK & $S \backslash\left\{S^{T h} \cap S^{m}, S^{F} \cap S^{a}\right\}$ \\
SE & $S \backslash\left\{S^{T u} \cap S^{e}\right\}$ \\
\hline &
\end{tabular}

Table A.10

Subsets of $G$ for the summer holiday cases.

\begin{tabular}{llll}
\hline Case & $G^{f}$ & $G^{l}$ & $G^{n}$ \\
\hline 1 & $\{2,3,8,12\}$ & $\{4,6,9,10,13\}$ & $\{1,5,7,11,14\}$ \\
2 & $\{2,5,8,9\}$ & $\{3,4,10,11,13\}$ & $\{1,6,7,12,14\}$ \\
3 & $\{2,5,6,7,8\}$ & $\{1,9,10,11,14\}$ & $\{3,4,12,13\}$ \\
\hline
\end{tabular}

of notation, we additionally introduce $S^{z}$ for $z \in\{M, T u, W, T h, F\}$, which denote all shifts on Monday, Tuesday, Wednesday, Thursday, and Friday, respectively. Then, Table A.9 gives $S N(t)$ for each task $t$. 
The subsets of gynecologists that are used in the summer holiday test cases are given in Table A.10.

\section{References}

[1] J. Van den Bergh, J. Beliën, P. De Bruecker, E. Demeulemeester, L. De Boeck, Personnel scheduling: a literature review, European J. Oper. Res. 226 (3) (2013) 367-385.

[2] P. De Bruecker, J. Van den Bergh, J. Beliën, E. Demeulemeester, Workforce planning incorporating skills: State of the art, European J. Oper. Res. 243 (1) (2015) 1-16.

[3] P. Smet, T. Wauters, M. Mihaylov, G. Vanden Berghe, The shift minimisation personnel task scheduling problem: A new hybrid approach and computational insights, Omega 46 (2014) 64-73.

[4] H. Beaulieu, J.A. Ferland, B. Gendron, M. Philippe, A mathematical programming approach for scheduling physicians in emergency room, Health Care Manage. Sci. 3 (2000) 193-200.

[5] R. Bruni, P. Detti, A flexible discrete optimization approach to the physician scheduling problem, Oper. Res. Health Care 3 (4) (2014) 191-199.

[6] C.A. White, G.M. White, Scheduling doctors for clinical training unit round using tabu optimization, in: E. Burke, P. De Causmaecker (Eds.), Practice and Theory of Automated Timetabling IV, in: Lecture Notes in Computer Science, vol. 2740, Springer, Berlin Heidelberg, 2003, pp. 120-128.

[7] A.C.C. Kreuk, E.M.M. De Winands, J.M.H. Vissers, Master Scheduling of Medical Specialists, Vol. 13, Beta, 2004.
[8] M. Hojati, A.S. Patil, An integer linear programming-based heuristic for scheduling heterogeneous, part-time service employees, European J. Oper. Res. 209 (1) (2011) 37-50.

[9] S. Bourdais, P. Galinier, G. Pesant, HIBISCUS: A constraint programming application to staff scheduling in health care, in: Principles and Practice of Constraint Programming, in: Lecture Notes in Computer Science, vol. 2833, 2003, pp. $153-167$.

[10] L.M. Rousseau, M. Gendreau, G. Pesant, A general approach to the physician rostering problems, Ann. Oper. Res. 115 (2002) 193-205.

[11] J. Li, E.K. Burke, T. Curtois, S. Petrovic, R. Qu, The falling tide algorithm: A new multi-objective approach for complex workforce scheduling, Omega 40 (3) (2012) 283-293.

[12] A. Gunawan, H.C. Lau, Master physician scheduling problem, J. Oper. Res. Soc. 64 (3) (2013) 410-425

[13] A. Fügener, J.O. Brunner, A. Podtschaske, Duty and workstation rostering considering preferences and fairness: a case study at a department of anaesthesiology, Int. J. Prod. Res. (2015).

[14] S.C. Ho, J.M.Y. Leung, Solving a manpower scheduling problem for airline catering using metaheuristics, European J. Oper. Res. 202 (3) (2010) 903-921.

[15] M. Erhard, J. Schoenfelder, A. Fügener, J.O. Brunner, State of the art in physician scheduling, European J.Oper. Res. 265 (1) (2018) 1-18.

[16] K.A. Dowsland, Nurse scheduling with tabu search and strategic oscillation, European J. Oper. Res. 106 (2-3) (1998) 393-407.

[17] P.J.M. van Laarhoven, E.H.L. Aarts, Simulated Annealing: Theory and Applications, in: Mathematics and Its Applications, vol. 37, Springer, Netherlands, 1987. 